\title{
Perfil sociodemográfico dos pacientes confirmados para Covid-19 residentes no Espírito Santo, Brasil
}

\section{Sociodemographic profile of patients confirmed with Covid-19 residing in Espírito Santo, Brazil}

\author{
André Diego da Silva Ferreira ${ }^{1}$, Leonardo Santos Perovano ${ }^{2}$, Lorrana Inacia Barboza ${ }^{3}$, Wilker Marcolino \\ Nascimento ${ }^{4}$, Franciele Montenegro da Silva ${ }^{5}$, Erika Cardoso dos Reis ${ }^{6}$ \\ ${ }^{1}$ Instituto Federal do Espírito Santo (IFES), Vitória, Espírito Santo, Brasil. ORCID: https://orcid.org/0000-0001-6736-6953 \\ 2 Instituto Federal do Espírito Santo (IFES), Vitória, Espírito Santo, Brasil. ORCID: https://orcid.org/0000-0003-3700-5588 \\ ${ }^{3}$ Instituto Federal do Espírito Santo (IFES), Vitória, Espírito Santo, Brasil. ORCID: https://orcid.org/0000-0001-5992-5356 \\ ${ }^{4}$ Instituto Federal do Espírito Santo (IFES), Vitória, Espírito Santo, Brasil. ORCID: https://orcid.org/0000-0001-5872-5491 \\ ${ }^{5}$ Instituto Federal do Espírito Santo (IFES), Vitória, Espírito Santo, Brasil. ORCID: https://orcid.org/0000-0002-5792-7718 \\ ${ }^{6}$ Instituto Federal do Espírito Santo (IFES), Vitória, Espírito Santo, Brasil. ORCID: https://orcid.org/0000-0003-4459-9345
}

Autor para correspondência/Mail to: André Diego da Silva Ferreira, andrediego85@gmail.com

Recebido/Submitted: 30 de agosto de 2020; Aceito/Approved: 03 de dezembro de 2020

Copyright (c) 2020 Ferreira, Perovano, Barboza, Nascimento, Silva \& Reis. Todo o conteúdo da Revista (incluindo-se instruções, política editorial e modelos) está sob uma licença Creative Commons Atribuição-NãoComercial-Compartilhalgual 3.0 Não Adaptada. Ao serem publicados por esta Revista, os artigos são de livre uso em ambientes educacionais, de pesquisa e não comerciais, com atribuição de autoria obrigatória. Mais informações em http://revistas.ufpr.br/atoz/about/submissions\#copyrightNotice.

\begin{abstract}
Resumo
Introdução: no final de 2019 o mundo foi acometido com o surgimento de uma doença, a Covid-19, que é causada pelo novo coronavírus SARS-COV-2, sendo inicialmente detectada na China e posteriormente se tornado uma pandemia, pois a doença ultrapassou várias fronteiras. Estudos deixam evidente que gênero, classe e raça apresentam condições de vulnerabilidade em relação à exposição da Covid-19, e estudos contribuem para a identificação dos grupos vulneráveis. Diante do exposto, realizou-se um estudo transversal de todos os casos confirmados com o objetivo de analisar as características sociodemográficas e a distribuição dos casos confirmados de Covid-19 nos municípios do Espírito Santo (ES). Metodologia: o estudo trata de uma pesquisa observacional transversal, que foi desenvolvida em duas etapas metodológicas. A primeira destina-se à obtenção da base de dados da Secretaria de Estado da Saúde do Espírito Santo (SESA) dos casos confirmados, realizada no dia 01/06/2020. A segunda etapa contemplou a recodificação, ordenação e distribuição das características sociodemográficas segundo região de saúde no Microsoft Excel e SPSS. Resultados: até 31/05/2020 foram registrados 13.386 casos confirmados da Covid-19 de residentes no ES. O perfil sociodemográfico predominante entre os pacientes é de pessoas pardas e pretas (39,4\%), com ensino médio completo ( $26,4 \%$ ), entre 20 e 39 anos (39,7\%) e do sexo feminino (54,1\%). Conclusão: com o passar dos três meses iniciais da pandemia de Covid-19, podemos constatar os impactos diferentes entre os grupos populacionais devido a vários fatores. Por tratar de uma doença que apresenta alta disseminação por contato, regiões populosas como a região metropolitana de Vitória-ES apresentam o maior número de casos dentro do ES.
\end{abstract}

Palavras-chave: Vírus SARS; Vigilância em Saúde Pública; Covid-19; Estudos Transversais.

\begin{abstract}
Introduction: at the end of 2019, the world was affected by the emergence of a disease, Covid-19, which is caused by the new coronavirus SARSCOV-2, being initially detected in China and later becoming a pandemic, as the disease crossed several borders. Studies made it clear that gender, class and race present conditions of vulnerability concerning Covid-19 exposure, and studies contribute to the identification of vulnerable groups. Given the above, it carries out a cross-sectional study of all confirmed cases to analyze the sociodemographic characteristics and the distribution of confirmed cases of Covid-19 in the municipalities of Espírito Santo (ES). Method: the study is a cross-sectional observational research, developed in two methodological steps. The first intends to obtain the database from the Secretary of State for Health of Espírito Santo (SESA) of confirmed cases carried out on 06/01/2020. The second stage included the recording, ordering and distribution of sociodemographic characteristics, statistical analysis of the information treated in Microsoft Excel and SPSS. Results: up to 05/31/2020, there were 13,386 confirmed cases of Covid-19 of residents in ES. The predominant sociodemographic profile among patients is brown and black (39.4\%), with complete secondary education (26.4\%), between 20 and 39 years old (39.7\%) and female (54.1\%). Conclusions: over the initial three months of the Covid-19 pandemic, we can see the different impacts among population groups due to several factors. As it deals with a disease that has high spread by contact, populous regions such as the metropolitan region of Vitória-ES have the largest number of cases within ES.
\end{abstract}

Keywords: SARS Virus; Public Health Surveillance; Covid-19; Cross-Sectional Studies.

\section{INTRODUÇÃO}

O final de 2019 ficou marcado com o surgimento da Covid-19, doença causada pelo novo coronavírus (SARSCoV-2), originalmente identificado na Cidade de Wuhan (China) (World Health Organization, 2020b) e que, posteriormente, no dia 11 de março, a Organização Mundial de Saúde (OMS) a classificou como uma pandemia, pois a distribuição espacial da doença ultrapassou várias fronteiras. Em virtude dessa elevada disseminação, tornou-se necessária a criação de um sistema para monitoramento dos casos da Covid-19 em tempo real no Brasil e no mundo (Cavalcante \& Abreu, 2020; Ludvigsson, 2020). 
A doença apresenta quadro clínico que varia de infecções assintomáticas a quadros respiratórios graves. Os sintomas aparecem após cinco ou seis dias após a infecção e os mais comuns são febres; tosse seca; dor de garganta; diarreia; ageusia, sendo caracterizada pela perda de paladar; anosmia, definida como a perda de olfato; e dispneia (Ahmed et al., 2020; Ng \& Tilg, 2020).

Uma característica do SARS-CoV-2, que contribuiu para que ele se tornasse um problema de saúde pública mundial, é sua alta taxa e potencial de transmissão, que ocorre por meio de objetos contaminados e de gotículas respiratórias de tosse e/ou de espirro (Cascella, Rajnik, Cuomo, Dulebohn, \& Di Napoli, 2020; Doremalen et al., 2020; World Health Organization, 2020a).

A Organização Pan-Americana de Saúde (OPAS), em meados de maio de 2020, demostrou que a doença acometeu mais de 6.1 milhões de pessoas, com uma taxa de mortalidade de 79 a cada 100.000 pessoas em todo o mundo (Organização Pan-Americana de Saúde, 2020). Segundo relatório publicado pela Organização Mundial de Saúde (OMS), os riscos devido à exposição, à suscetibilidade biológica, às implicações econômicas e outros determinantes sociais variam entre os indivíduos em relação à infecção (Nepomuceno et al., 2020; World Health Organization, 2020b).

Conforme Drefahl et al. (2020), análises rudimentares dos casos detectados na Suécia demonstraram que o número absoluto de pessoas contaminadas pela Covid-19 varia em proporções distintas nos diversos setores da sociedade. Esses resultados sugerem que homens, idosos, pessoas de classes étnicas consideradas minoritárias e pessoas que ocupam baixas posições sociais são os mais suscetíveis a desenvolver um quadro grave da doença (Aldridge et al., 2020; Cook, Kursumovic, \& Lennane, 2020; Dowd et al., 2020; Du et al., 2020; Jin et al., 2020; Lippi, Mattiuzzi, Sanchis-Gomar, \& Henry, 2020; Lusignan et al., 2020; Onder, Rezza, \& Brusaferro, 2020; Ruan, Yang, Wang, Jiang, \& Song, 2020; Williamson et al., 2020; Zhang et al., 2020).

Os dados coletados pelo Departamento de Saúde da cidade de Nova York, publicados no dia 2 de abril pelo jornal The New York Times, demonstram que os bairros de baixa renda estão sendo mais atingidos pela epidemia do Covid-19 naquela cidade, não apenas com um número maior de casos confirmados, mas também de casos mais severos. Isso significa que além de estarem mais sujeitos à contaminação, os moradores dessa região estão desenvolvendo quadros mais graves da doença (Pires, Carvalho, \& Xavier, 2020; Sousa et al., 2020).

Em contraste ao sistema de saúde dos Estados Unidos, os países que contêm sistemas universais de saúde, como o Sistema Único de Saúde (SUS), em teoria devem conter menos casos de infecção e mortalidade advindos da Covid-19 (Aquino et al., 2020; Maciel, E. L. et al., 2020).

Nesse cenário, considerando-se os atuais níveis de desigualdade social e o grande número de pessoas em situação de vulnerabilidade sociodemográfica no Brasil, a quantidade de casos da Covid-19 pode apresentar distinções nas progressões das taxas de incidência e mortalidade, sendo associados às doenças preexistentes que, em locais onde se encontram as maiores desigualdades, são facilmente identificadas, devido à dificuldade de acesso aos procedimentos médicos (Chiriboga, Garay, Buss, S., \& Rispel, 2020; Demenech, Dumith, Vieira, \& Neiva-Silva, 2020; Moraes Filho et al., 2020).

A vulnerabilidade social resulta na desproporcionalidade da exposição aos riscos sociais e de saúde, além de influenciar na capacidade de resposta e recuperação da comunidade (Kim \& Bostwick, 2020).

Estudos apresentados por Estrela et al. (2020b) e Estrela et al. (2020a) deixam evidente que gênero, classe e raça apresentam condições de vulnerabilidade em relação à exposição da Covid-19, assim como Miller et al. (2020) que, em seu estudo realizado na cidade de Detroit, nos Estados Unidos, verificaram as disparidades raciais entre negros, latinos e brancos.

Esses fatores sociais que podem contribuir com condição de vulnerabilidade permanecem com poucos estudos, principalmente em cidades com baixo status socioeconômico. Desta forma registros e estudos desses fatores, como raça/etnia dos pacientes, contribui para a identificação dos grupos vulneráveis (Khalatbari-Soltani, Cumming, Delpierre, \& Kelly-Irving, 2020).

A Fiocruz emitiu, em 23 de março de 2020, um relatório que classifica as microrregiões brasileiras com alta probabilidade de epidemia a curto prazo, e que também possuem alta vulnerabilidade social. Segundo o documento, a região Sudeste, a qual o estado do Espirito Santo pertence, tem grande probabilidade de acumular casos devido à grande quantidade de pessoas em risco e a conectividade com São Paulo e Rio de Janeiro, que eram naquela data (23/03) dois epicentros da doença no Brasil (Codeço et al., 2020).

Segundo o boletim epidemiológico especial feito pelo Centro de Operações de Emergências em Saúde Pública (COE-COVID-19) publicado pelo Ministério da Saúde (MS) em 31/05/2020, data que foi realizado o estudo, o Brasil possuía 526.447 mil casos confirmados e o Espírito Santo, objeto de estudo, contava 13.690 casos confirmados (Ministério da Saúde, 2020).

Diante do exposto, este artigo tem como objetivo analisar as características sociodemográficas e a distribuição dos casos confirmados de Covid-19, sendo eles sexo, faixa etária, raça e escolaridade para os municípios do Espírito Santo, segundo os dados disponibilizados pela Secretaria Estadual de Saúde do Espírito Santo (SESA). 


\section{PROCEDIMENTOS METODOLÓGICOS}

Trata-se de uma pesquisa observacional, descritiva, transversal que avaliou todos os casos confirmados de Covid-19 no estado do Espírito Santo (ES) registrados até 31 de maio de 2020.

O estudo envolveu duas etapas metodológicas. A primeira destina-se à obtenção da base de dados, que foi extraída do painel público no site eletrônico www.coronavirus.es.gov.br, gerenciado pela Secretaria de Saúde do Estado do Espírito Santo (SESA), dos casos confirmados de doença pelo coronavírus (SARS-CoV-2), que foi realizada no dia 01/06/2020. No momento da realização deste estudo, o ES ocupava a $2^{\circ}$ posição no Índice de Transparência da Covid-19 segundo a ONG Open Knowledge Brasil (OKBR).

A segunda etapa contemplou a ordenação e análise estatística das informações obtidas na etapa anterior. Os municípios foram agrupados segundo o vigente Plano Diretor de Regionalização da Saúde do Espírito Santo de 2011, o qual relata que a divisão deve considerar as características culturais, socioeconômicas e os aspectos políticos interfederativos, na medida em que o funcionamento do sistema regional é fruto da pactuação entre os gestores do sistema de saúde. Sendo assim, os municípios foram agrupados em quatro Regiões de Saúde, sendo elas: Norte, Metropolitana, Sul e Central. Foram excluídos todos os pacientes não residentes no estado do Espírito Santo.

Foram analisadas as variáveis (I) faixa etária, que foi categorizada em: 0 a 9 anos; 10 a 19 anos; 20 a 39 anos; 40 a 59 anos; e 60 anos ou mais. Além dessas, (II) raça/cor, categorizada em: amarelos, brancos, indígenas, pretos/pardos e ignorado; (III) sexo: masculino e feminino; e (IV) escolaridade, categorizada em: ensino superior, ensino médio completo, ensino fundamental completo, ensino fundamental incompleto e analfabeto, em relação à região de saúde. A organização e análise dos dados foram realizadas no Microsoft Excel e $\operatorname{SPSS} \mathbb{R}($ Statistical Package for Social Sciences) versão 25.

Foi calculada a frequência absoluta e relativa de todas as variáveis contidas no presente estudo, e foi verificada a distribuição das características sociodemográficas, a saber: escolaridade, faixa etária, sexo raça/cor e região de saúde de residência. A seleção das variáveis para análise se deu em função da variabilidade sociodemográfica das regiões de saúde do ES, com municípios predominantemente urbanos e com acesso mais ampliado a bens e serviços de saúde na região metropolitana, e municípios com características mais rurais nas demais regiões do estado.

O estudo foi realizado conforme os princípios éticos que constam na Resolução 466/2012 e 512/2016 do Conselho Nacional de Saúde e, por utilizar uma base de dados de acesso livre e sem identificação dos pacientes, não foi necessária a aprovação do trabalho no Comitê de Ética em Pesquisa.

\section{RESULTADOS E DISCUSSÕES}

Até 31/05/2020 foram registrados 13.386 casos confirmados da Covid-19 de residentes no Espírito Santo. O perfil sociodemográfico predominante entre os pacientes dessa doença é de pessoas pardas e pretas (39,4\%), com ensino médio completo $(26,4 \%)$, entre 20 e 39 anos $(39,7 \%)$ e do sexo feminino $(54,1 \%)$, conforme a Tabela 1.

\begin{tabular}{lcc}
\hline Variáveis & $\mathbf{n}$ & Pacientes \\
& & \\
\hline Sexo & 7234 & \\
\hline Feminino & 6149 & 54,1 \\
Masculino & & 45,9 \\
\hline Raça/cor & 763 & \\
\hline Amarela & 4321 & 5,7 \\
Branca & 3002 & 32,3 \\
Ignorado & 16 & 22,4 \\
Indígena & 5284 & 0,1 \\
Preta/Parda & & 39,4 \\
\hline Escolaridade & 1393 & \\
\hline Ensino fundamental incompleto & 1068 & 16 \\
Ensino fundamental completo & 198 & 2,3 \\
Analfabeto & 2200 & 25,2 \\
Educação superior & 3858 & 44,2 \\
Ensino médio completo & & \\
\hline
\end{tabular}




\begin{tabular}{lcc}
\hline Variáveis & $\mathbf{n}$ & Pacientes \\
& & \\
\hline Faixa etária & 197 & \\
\hline Oa 9 anos & 304 & 1,5 \\
10 a 19 anos & 5317 & 2,3 \\
20 a 39 anos & 5176 & 39,7 \\
40 a 59 anos & 2392 & 38,7 \\
60 ou mais & & 17,9 \\
\hline Região de saúde de residência & 557 & \\
\hline Região Norte & 982 & 7,2 \\
Região Central & 10470 & 78,2 \\
Região Metropolitana & 1377 & 10,3 \\
Região Sul & & \\
\hline
\end{tabular}

Tabela 1. Características sociodemográficas dos casos confirmados COVID-19 no Espírito Santo, 2020. Fonte: Elaborado pelos autores a partir de dados disponibilizados pela SESA (2020).

Com relação aos sexos dos casos registrados, percebe-se uma distribuição relativamente uniforme entre mulheres $(54,1 \%)$ e homens $(45,9 \%)$, com variações entre os grupos de idade. Os resultados encontrados diferem dos dados da OMS que, ao apresentar a distribuição mundial do sexo entre os casos confirmados, apontou maior proporção de mulheres $(47 \%)$ do que homens (51\%) (World Health Organization, 2020a).

Segundo Chen et al. (2020), a Covid-19 parece ter uma preferência entre gêneros. No estudo, observou-se a maior parte dos casos está presente no sexo masculino. Em pandemias anteriores causadas pelos vírus do gênero SARS-CoV e MERS-Cov (MERS é a sigla em inglês de Síndrome Respiratória do Oriente Médio), os homens eram mais propensos a serem infectados do que as mulheres.

Isso pode ter relação com o fato de que, embora mais susceptíveis à contaminação por coronavírus, os homens foram mais negligentes e não realizaram quarentena de forma voluntária. Refletindo no paradigma social imaginário, na qual o homem se vê como um ser invulnerável, o que contribui para que se cuide menos e se exponha mais a situações de risco (Gomes, Nascimento, \& Araújo, 2020).

Conforme Lopes e Leal (2005), uma possibilidade de as mulheres apresentarem maior quantidade de casos talvez seja devido ao fato de as análises terem incluído profissionais de saúde, visto que, no setor de saúde, a mão de obra é predominantemente feminina e mais exposta aos riscos.

Outra possibilidade, segundo os dados do último censo demográfico realizado pelo Instituto Brasileiro de Geografia e Estatística (IBGE), no ano de 2010, é que a população do ES apresentou em maioria as mulheres (1,78 milhão) em relação aos homens (1,73 milhão) (Instituto Jones dos Santos Neves, 2011). Dessa forma, como a distribuição populacional tem maiores números absolutos de mulheres, elas podem ser mais infectadas.

Com relação à faixa etária, no presente estudo o grupo de 20-39 teve o maior percentual de casos de coronavírus $(39,7 \%)$ e pessoas acima de 40 anos apresentam aproximadamente $57 \%$ do total de casos. Comparando com os resultados apresentados por (Cheng \& Shan, 2020), o grupo de 20-39 conteve o maior risco de contaminação a Covid-19, sendo que $72 \%$ dos casos confirmados tem idade igual ou superior a 40 anos e, com base nos resultados, percebe-se que os dados não divergem do estudo.

Lima et al. (2020) ressaltam que os idosos constituem um fator de extrema preocupação, visto que o aumento da idade está atrelado ao aumento da mortalidade. Adicionalmente, o estudo relacionou que os idosos possuem crendices que a pandemia no Brasil será menor do que a registrada em outros países e que possuem maior proteção ao novo coronavírus.

Dessa forma, os dados apontam a maior incidência nos grupos maiores de 20 anos de idade à contaminação por Covid-19, mesmo resultado apontado por Gouveia et al. (2020) que, na cidade do Ceará, apresentou predominância da faixa etária entre 20 e 59 anos.

O nível de escolaridade aponta que aproximadamente $70 \%$ dos casos possuem no mínimo o ensino médio. Contrapondo a este fato, Lenzi, Wiens, Grochocki, e Pontarolo (2020) demonstram que a escolaridade é um fator de risco para disseminação de doenças virais infecciosas. Tendo em vista que esse fator pode se relacionar com a classe social do indivíduo, sugerindo que os hábitos, as condições de vida e o conhecimento sobre a doença influenciam no prognóstico. Desta forma, indivíduos com escolaridade mais baixa estariam mais propensos a contrair a infecção, pois utilizam o transporte público, moram e frequentam locais com maior número de indivíduos e têm menos acesso a recursos médicos.

Com base nesse preceito, ressalta-se a importância do nível de escolaridade, que traz consigo importantes descrições sociais dos casos, servindo como forma de identificação e prevenção à disseminação da doença. 
O estudo demonstrou que, nesse período avaliado, as pessoas que foram acometidas pela doença são pretos / pardos $(39,4 \%)$, sendo sucedido pelos brancos $(32,3 \%)$ e os menos atingidos são os indígenas $(0,1 \%)$. Vale a pena ressaltar que no ES, segundo o boletim semanal publicado pela Articulação dos Povos Indígenas do Brasil (APIB), há casos das doenças no povo indígena Tupiniqim (Articulação dos Povos Indígenas do Brasil, 2020).

Conforme Santos et al. (2020), a população parda/negra tem $62 \%$ a mais de risco de contrair a doença, visto que, as diferenças de saúde entre os grupos estão relacionadas aos fatores econômicos e sociais, sendo percebidos nos recursos que cada grupo pode adquirir para sua proteção.

De acordo com Vallat e Wilson (2003), os impactos socioeconômicos causados por enfermidades como a Covid-19 geram aumento de pobreza, pois quem não possui acesso a condições dignas de moradia e vida necessita se expor, tornando-se cada vez mais vulnerável. Uma possibilidade de explicação demonstrada por Bhala, Curry, Martineau, Agyemang, e Bhopal (2020) é que pessoas pardas/pretas trabalham em setores essenciais na saúde e atendimento social, varejistas, transporte público e outros setores, colocando-se na linha de frente e consequentemente com risco de exposição à Covid-19.

A região metropolitana contém maior predominância dos casos registrados em todas as categorias sociodemográficas analisadas, tendo valores acima de $60 \%$ dos casos. As regiões sul, norte e central sucedem a região metropolitana na respectiva ordem.

\section{Região de Saúde}

\begin{tabular}{lcccccccc} 
Variáveis & Norte & \multicolumn{3}{c}{ Central } & \multicolumn{3}{c}{ Metropolitana } & Sul \\
\hline & $\mathbf{n}$ & $\%$ & $\mathbf{n}$ & $\%$ & $\mathbf{n}$ & $\%$ & $\mathbf{n}$ & $\%$ \\
\hline Escolaridade & & & & & & & & \\
\hline Ensino superior & 121 & 5,5 & 173 & 7,9 & 1680 & 76,4 & 226 & 10 \\
Ensino médio completo & 176 & 4,6 & 303 & 7,9 & 2987 & 77,4 & 392 & 10 \\
Ensino fundamental completo & 53 & 5 & 109 & 10,2 & 770 & 72,1 & 136 & 13 \\
Ensino fundamental incompleto & 86 & 6,2 & 160 & 11,5 & 898 & 64,5 & 249 & 18 \\
Analfabeto & 20 & 10 & 16 & 8,1 & 133 & 67,2 & 29 & 15 \\
\hline Faixa Etária & & & & & & & & \\
\hline 60 anos ou mais & 86 & 3,6 & 147 & 6,1 & 1932 & 80,8 & 227 & 9,5 \\
40-59 anos & 212 & 4,1 & 372 & 7,2 & 4056 & 78,4 & 536 & 10 \\
20-39 anos & 211 & 4 & 406 & 7,6 & 4159 & 78,2 & 541 & 10 \\
10-19 anos & 29 & 9,5 & 38 & 12,5 & 200 & 65,8 & 37 & 12 \\
0-9 anos & 19 & 9,6 & 19 & 9,6 & 123 & 62,4 & 36 & 18 \\
\hline Sexo & & & & & & & & \\
\hline Masculino & 253 & 4,1 & 464 & 7,5 & 4780 & 77,7 & 652 & 11 \\
Feminino & 304 & 4,2 & 518 & 7,2 & 5687 & 78,6 & 725 & 10 \\
\hline Raça/Cor & & & & & & & & \\
\hline Preta/Pardo & 276 & 9 & 389 & 11,9 & 3533 & 64,3 & 428 & 15 \\
Indígena & 0 & 0 & 4 & 25 & 10 & 62,5 & 2 & 13 \\
Branca & 166 & 3,8 & 406 & 9,4 & 2986 & 69,1 & 763 & 18 \\
Amarela & 70 & 9,2 & 93 & 12,2 & 540 & 70,8 & 60 & 7,9 \\
\hline
\end{tabular}

Tabela 2. Distribuição das características sociodemográficas por regiões de saúde dos casos confirmados de Covid-19 no Espírito Santo, 2020. Fonte: Elaborado pelos autores a partir de dados disponibilizados pela SESA (2020).

Com relação às faixas etárias, observou-se que pessoas com mais de 60 anos representam aproximadamente metade dos casos de contaminação por Covid-19 para a região metropolitana de saúde enquanto o grupo mais afetado é o grupo entre 20-39 anos. Esse fato foi descrito por Sousa et al. (2020) em que a chegada do coronavírus no Brasil deu-se através de pessoas que estiveram no exterior e iniciou-se pelas grandes capitais, dessa forma era de se esperar no momento do estudo que as populações da região metropolitana tivessem mais chances de contato direto com alguém testado positivamente para o coronavírus comparado aos que moram no interior.

Um fato deveras importante citado por Vargas (2016) é que a vida mais interiorana proporciona uma maior teia de suporte social, ajudando na sobrevivência, suprindo a própria ausência do estado nas suas muitas necessidades. Essa situação cria vínculos que podem tornar a distância e o isolamento mais difíceis. Provavelmente, esses vínculos e a ligação social mais próxima em cidades do interior sirvam de fortalecimento de determinadas crenças.

Conforme esse preceito apresentado, ressalta-se a importância das medidas preventivas tomadas pela vigilância em saúde, pois o isolamento provocado nos centros urbanos é considerado mais simples do que nas regiões interioranas, assim, a localização geográfica dos epicentros da Covid-19 pode mudar dos grandes centros urbanos para o interior. 
Avaliando a raça/cor, percebe-se que em todas as regiões de saúde a raça/cor branca e preta/parda contêm as maiores taxas de quantidade de casos. Esse fator pode estar associado aos demais apresentados como as características econômicas e sociais.

Com relação ao nível de escolaridade, nota-se que a maior parte dos casos registrados em todas as regiões são pessoas com alto nível de escolaridade. O estudo publicado por Lima et al. (2020) demonstra que, na associação entre as respostas do questionário e o nível de escolaridade, os participantes com o ensino fundamental consideraram que estão em um nível de risco menos alto que os participantes com grau de escolaridade mais elevado. As pessoas com ensino fundamental também creem que a situação sanitária em que vive a maioria da população mais pobre fará com que a contaminação pela Covid-19 seja menor que na população de alta renda.

\section{CONCLUSÃO}

Com o passar dos três meses iniciais da doença e, consequentemente, a pandemia de Covid-19, podemos constatar os impactos diferentes entre os grupos populacionais devido à suscetibilidade biológica, social e educacional. Por se tratar de uma doença que apresenta alta disseminação por contato, regiões populosas como a região metropolitana de Vitória (ES) apresentam o maior número de casos dentro do ES. Porém, vale ressaltar que as cidades do interior também devem se atentar com a escalada de números de casos da Covid-19, porque diferentemente das regiões metropolitanas, as coberturas hospitalares dessas cidades são deficitárias.

A análise da distribuição de fatores sociodemográficos possibilita a determinação de perfis com maior vulnerabilidade, o que faz deste estudo relevante, uma vez que gera informações para as autoridades competentes conhecerem os perfis dos grupos populacionais do Estado e assim planejarem uma cobertura assistencial, de forma mais eficiente, a fim de conter o contágio comunitário em nossa população. Essa prioridade de recursos para o grupo com maior vulnerabilidade torna-se importante passo nesse momento, pois agindo de forma coordenada podemos frear a evolução dos números de casos e consequentemente dos números de óbitos.

\section{AGRADECIMENTOS}

A professora Erika Cardoso dos Reis, mestre e doutora em saúde pública (ENSP/FIOCRUZ) pela oportunidade de estarmos desenvolvendo essa atividade de complemento ao ensino.

A Secretaria de Saúde do Estado do Espírito Santo pela disponibilização dos dados no endereço coronavirus.es.gov.br, sendo categorizados conforme os fatores sociodemográficos.

A todos os envolvidos direta e indiretamente nessa publicação. 


\section{REFERÊNCIAS}

Ahmed, W., Angel, N., Edson, J., Bibby, K., Bivins, A., O'Briend, J. W., ... Mueller, J. F. (2020). First confirmed detection of sars-cov-2 in untreated wastewater in australia: A proof of concept for the wastewater surveillance of covid19 in the community. Science of the Total Environment, 728, 138764. doi: 10.1016/j.scitotenv.2020.138764.

Aldridge, R. W., Lewer, D., Katikireddi, S. V., Mathur, R., Pathak, N., Bruns, R., ... Hayward, A. (2020). Black, asian and minority ethnic groups in england are at increased risk of death from covid-19: indirect standardisation of nhs mortality data. Wellcome Open Research, 5(88). doi: 10.12688/wellcomeopenres.15922.2.

Aquino, E. M. L., Silveira, I. H., Pescarini, J. M., Aquino, R., Souza-Filho, J. A. d., Rocha, A. d. S., ... Lima, R. T. d. R. S. (2020). Medidas de distanciamento social no controle da pandemia de covid-19: potenciais impactos e desafios no brasil. Ciência ES Saúde Coletiva, 25(supp 1). doi: 10.1590/141381232020256.1.10502020.

Articulação dos Povos Indígenas do Brasil. (2020). Vidas indígenas e o covid-19. Recuperado de http:// emergenciaindigena.apib.info/dados_covid19/.

Bhala, N., Curry, G., Martineau, A. R., Agyemang, C., \& Bhopal, R. (2020). Sharpening the global focus on ethnicity and race in the time of covid-19. The Lancet, 395 (10238), 1673-1676. doi: 10.1016/S0140-6736(20)31102-8.

Cascella, M., Rajnik, M., Cuomo, A., Dulebohn, S., \& Di Napoli, R. (2020). Features, evaluation and treatment coronavirus (covid-19). Treasure Island, FL: StatPearls Publishing.

Cavalcante, J. R., \& Abreu, A. J. L. (2020). Covid-19 no município do rio de janeiro: análise espacial da ocorrência dos primeiros casos e óbitos confirmados. Eidemiologia e Serviços de Saúde, 29(3), 1-10. doi: 10.5123/s167949742020000300007.

Chen, N., Zhou, M., Dong, X., Qu, J., Gong, F., Han, Y., ... Zhang, L. (2020). Epidemiological and clinical characteristics of 99 cases of 2019 novel coronavirus pneumonia in wuhan, china: a descriptive study. The Lancet, 395 (10223), 1690-1691. doi: 10.1016/S0140-6736(20)30211-7.

Cheng, Z. J., \& Shan, J. (2020). Novel coronavirus: where we are and what we know. Infection, 48(2), 155-163. doi: 10.1007/s15010-020-01401-y.

Chiriboga, D., Garay, J., Buss, P., S., M. R., \& Rispel, L. C. (2020). Health inequity during the covid-19 pandemic: a cry for ethical global leadership. The Lancet, 395(10238), 1690-1691. doi: 10.1016S0140-6736(20)31145-4.

Codeço, C. T., Villela, D., Coelho, F., Bastos, L. S., Carvalho, L. M., Gomes, M. F. C., ... Lana, R. M. (2020). Estimativa de risco de espalhamento da covid-19 no brasil e avaliação da vulnerabilidade socioeconômica nas microrregiões brasileiras. 2. relatório - 23 de março de 2020. Recuperado de https://www.arca.fiocruz.br/bitstream/ icict/40509/4/Relatorio_tecnico_COVID-19_procc-emap -covid-19-reporte2_resultados.pdf.

Cook, T., Kursumovic, E., \& Lennane, S. (2020). Exclusive: deaths of nhs staff from covid-19 analysed. Recuperado de https://www.hsj.co.uk/exclusive-deaths-of-nhs -staff-from-covid-19-analysed/7027471.article.

Demenech, L. M., Dumith, S. C., Vieira, M. E. C. D., \& Neiva-Silva, L. (2020). Desigualdade econômica e risco de infecção e morte por covid-19 no brasil. Revista Bra- sileira de Epidemiologia, 23, e200095. doi: 10.1590/1980549720200095.

Doremalen, N. v., Bushmaker, T., Morris, D. H., Holbrook, M. G., Gamble, A., Brandi, B. N., N. Williamson, ... Munster, V. J. (2020). Aerosol and surface stability of sars-cov-2 as compared with sars-cov-1. New England Journal of Medicine, 382(16), 1564-1567. doi: 10.1056/NEJMc2004973.

Dowd, J. B., Andriano, L., M., B. D., Rotondi, V., Block, P., Ding, X., ... Mills, M. C. (2020). Demographic science aids in understanding the spread and fatality rates of covid19. Proceedings of the National Academy of Sciences of the United States of America, 117(18), 9696-9698. doi: 10.1073/pnas.2004911117.

Drefahl, S., Wallace, M., Mussino, E., Aradhya, S., Kolk, M., Brandén, M., ... Andersson, G. (2020). A population-based cohort study of socio-demographic risk factors for covid-19 deaths in sweden. Nature Communications, 11(5097). doi: 10.1038/s41467-020-18926-3.

Du, R.-H., Liang, L.-R., Yang, C.-Q., Wang, W., Cao, T.-Z., Li, M., ... Zhong Shi, H.-Z. (2020). Predictors of mortality for patients with covid-19 pneumonia caused by sars-cov-2: a prospective cohort study. European Respiratory Journal, 55(5), 2000524. doi: 10.1183/13993003.00524-2020.

Estrela, F. M., Soares e Soares, C. F., Cruz, M. A., Silva, A. F. d., Santos, J. R. L., Moreira, T. M. d. O., ... Silva, M. G. (2020a). A model of disparities: risk factors associated with covid-19 infection. International Journal for Equity Health, 19(1), 1-10. doi: 10.1186/s12939-020-01242-z.

Estrela, F. M., Soares e Soares, C. F., Cruz, M. A., Silva, A. F. d., Santos, J. R. L., Moreira, T. M. d. O., .. Silva, M. G. (2020b). Pandemia da covid 19: refletindo as vulnerabilidades a luz do gênero, raça e classe. Ciência E Saúde Coletiva, 25(9), 3431-3436. doi: 10.1590/141381232020259.14052020 .

Gomes, R., Nascimento, E. F., \& Araújo, F. C. (2020). Por que os homens buscam menos os serviços de saúde do que as mulheres? as explicações de homens com baixa escolaridade e homens com ensino superior. Cadernos de Saúde Pública, 23(3), 565-574. doi: 10.1590/S0102-311X2007000300015.

Gouveia, G. P. M., Gouveia, S. S., Vasconcelos, S. S., Silva, E. d. S. M., Medeiros, R. G., \& Silva, R. L. d. (2020). Incidência e letalidade da covid-19 no ceará, 2020. Cadernos ESP/CE , 14(1), 10-16. Recuperado de https://cadernos .esp.ce.gov.br/index.php/cadernos/article/view/326.

Instituto Jones dos Santos Neves. (2011). Distribuição populacional no espírito santo: resultados do censo demográfico 2010. IJSN , 4 (27), 1-11. Recuperado de http://www.ijsn .es.gov.br/ConteudoDigital/20121022_958_201127_.pdf.

Jin, J.-M., Bai1, P., He, W., Wu, F., Liu, X.-F., Han, D.-M., ... Yang, J.-K. (2020). Gender differences in patients with covid-19: focus on severity and mortality. Frontiers in Public Health, 55 (5), 2000524. doi: 10.1183/13993003.00524-2020.

Khalatbari-Soltani, S., Cumming, R. C., Delpierre, C., \& Kelly-Irving, M. (2020). Importance of collecting data on socioeconomic determinants from the early stage of the covid-19 outbreak onwards. Journal of Epidemiology and Community Health, 74(8), 620-623. doi: 10.1136/jech2020-214297.

Kim, J. S., \& Bostwick, W. (2020). Social vulnerability and racial inequality in covid-19 deaths in chicago. Revista Brasileira de Epidemiologia, 47(4), 509-513. doi: $10.1177 / 1090198120929677$.

Lenzi, L., Wiens, A., Grochocki, M. H., \& Pontarolo, R. 
(2020). Study of the relationship between socio-demographic characteristics and new influenza a (h1n1). Brazilian Journal fo Infection Disease, 15(5), 457-461. doi: 10.1590/S141386702011000500007.

Lima, D. L. F., Dias, A. A., Rabelo, R. S., Cruz, I. D. d., Costa, S. C., Nigri, F. M. N., \& Neri, J. R. (2020). Covid-19 no estado do ceará, brasil: comportamentos e crenças na chegada da pandemia. Ciência Es Saúde Coletiva, 25(5), 1575-1586. doi: 10.1590/1413-81232020255.07192020.

Lippi, G., Mattiuzzi, C., Sanchis-Gomar, F., \& Henry, B. M. (2020). Clinical and demographic characteristics of patients dying from covid-19 in italy vs china. Journal of Medical Virology, 92(10). doi: 10.1002/jmv.25860.

Lopes, M. J. M., \& Leal, S. M. C. (2005). A feminização persistente na qualificação profissional da enfermagem brasileira. Cadernos Pagu(24), 105-125. doi: 10.1590/S010483332005000100006.

Ludvigsson, J. F. (2020). Systematic review of covid19 in children shows milder cases and a better prognosis than adults. Acta Paediatrica, 109(6), 1088-1095. doi: 10.1111/apa.15270.

Lusignan, S. d., Dorward, J., Correa, A., Jones, N., Akinyemi, O., Amirthalingam, G., ... Richard Hobbs, F. D. R. (2020). Risk factors for sars-cov-2 among patients in the oxford royal college of general practitioners research and surveillance centre primary care network: a cross-sectional study. Lancet Infections Disease, 20(9), 1034-1042. doi: 10.1016/S1473-3099(20)30371-6.

Maciel, E. L. et al. (2020). Fatores associados ao óbito hospitalar por covid-19 no espírito santo, 2020. Epidemiologia e Serviços de Saúde, 29(4), 1-11. doi: 10.1590/s167949742020000400022.

Miller, J., Fadel, R. A., Tang, A., Perrotta, G., Herc, E., Soman, S., . . Suleyman, G. (2020). The impact of sociodemographic factors, comorbidities, and physiologic responses on 30-day mortality in coronavirus disease 2019 (covid-19) patients in metropolitan detroit. Clinical Infectious Diseases(ciaa1420). doi: 10.1093/cid/ciaa1420.

Ministério da Saúde. (2020). Coronavírus (covid-19). Recuperado de https://coronavirus.saude.gov.br/sobre-a-doenca

Moraes Filho, I. M., Sousa, T. V. d., Carvalho Filha, F. S. S., Pereira, M. C., Vilanova, J. d. M., \& Silva, R. M. d. (2020). Fatores sociodemográficos e emocionais associados à tolerância nas relações de amizade na pandemia pela covid19. Revista de enfermagem da UFSM, 11, 1-17. doi: $10.5902 / 2179769253180$.

Nepomuceno, M. R., Acosta, E., Alburez-Gutierrez, D., Aburto, J. M., Gagnon, A., \& Turra, C. M. (2020). Besides population age structure, health and other demographic factors can contribute to understanding the covid-19 burden. Proceedings of the National Academy of Sciences of the United States of America, 117(25), 13881-13883. doi: $10.1073 /$ pnas. 2008760117 .

Ng, S. C., \& Tilg, H. (2020). Covid-19 and the gastrointestinal tract: more than meets the eye. Gut, 69(9), 973-974. doi: 10.1136/gutjnl-2020-321195.

Onder, G., Rezza, G., \& Brusaferro, S. (2020). Casefatality rate and characteristics of patients dying in relation to covid-19 in italy. JAMA, 323(18), 1775-1776. doi: 10.1001/jama.2020.4683.

Organização Pan-Americana de Saúde. (2020). Folha informativa covid-19 - escritório da opas e da oms no brasil.
Recuperado de https://www.paho.org/pt/covid19.

Pires, L. L., Carvalho, L., \& Xavier, L. L. (2020). Covid-19 e desigualdade no brasil. doi: 10.13140/RG.2.2.27014.73282.

Ruan, Q., Yang, K., Wang, W., Jiang, L., \& Song, J. (2020). Clinical predictors of mortality due to covid-19 based on an analysis of data of 150 patients from wuhan, china. JAMA, 46(5), 1775-1776. doi: 10.1001/jama.2020.4683.

Santos, M. P. A. d., Neryi, J. S., Goes, E. F., Silva, A. d., Santos, A. B. S., Batista, L. E., \& Araújo, E. M. d. (2020). População negra e covid-19: reflexões sobre racismo e saúde. Estudos Avançados, 34(99), 225-243. doi: 10.1590/s01034014.2020.3499.014.

Sousa, G. J. B., Garces, T. S., Cestari, V. R. F., Moreira, T. M. M., Florêncio, R. S., \& Pereira, M. L. D. (2020). Estimação e predição dos casos de covid-19 nas metrópoles brasileiras. Revista Latino-Americana de Enfermagem, 28(e3345). doi: 10.1590/1518-8345.4501.3345.

Vallat, B., \& Wilson, D. (2003). Les obligations des pays membres de l'oie (organisation mondiale de la santé animale) en matière d'organisation de leurs services vétérinaires. Revue Scientifique et Technique, 22, 553-559.

Vargas, M. A. R. (2016). Moradia e pertencimento: a defesa do lugar de viver e morar por grupos sociais em processo de vulnerabilização. Cadernos Metrôpole, 18(36), 535-558. doi: 10.1590/2236-9996.2016-3611.

Williamson, E. J., Walker, A. J., Bhaskaran, K., Bacon, S., Bates, C., Morton, C. E., ... Goldacre, B. (2020). Factors associated with covid-19-related death using opensafely. $\mathrm{Na}$ ture, 584 (7821), 430-436. doi: 10.1038/s41586-020-2521-4.

World Health Organization. (2020a). Gender and covid19: Advocacy brief. Recuperado de https://www.who.int/ publications/i/item/gender-and-covid-19.

World Health Organization. (2020b). Origin of sars-cov-2. Recuperado de https://www.who.int/publications-detail/ origin-of-sars-cov-2.

Zhang, J., Wang, X., Jia, X., Li, J., Hu, K., Chen, G., ... Dong, W. (2020). Risk factors for disease severity, unimprovement, and mortality in covid-19 patients in wuhan, china. Clinical Microbiology and Infection, 26(6), 767-772. doi: 10.1016/j.cmi.2020.04.012.

Ferreira, A. D. da S., Perovano, L. S., Barboza, L. I., Nascimento, W. N., Silva, F. M. da \& Reis, E. C. dos (2020). Perfil sociodemográfico dos pacientes confirmados para Covid-19 residentes no Espírito Santo, Brasil. AtoZ: novas práticas em informação e conhecimento, 9(2), 216 - 223. Recuperado de: http:// dx.doi.org/10.5380/atoz.v9i2.76179 